\title{
Transcriptional Trajectories in Mouse Limb Buds Reveal the Transition from Anterior-Posterior to Proximal-Distal Patterning at Early Limb Bud Stage
}

\author{
Ines Desanlis ${ }^{1,2}$, Rachel Paul ${ }^{1,3}$ and Marie Kmita 1,2,3,* \\ 1 Genetics and Development Research Unit, Institut de Recherches Cliniques de Montréal, \\ Montreal, QC H2W 1R7, Canada; Ines.Desanlis@ircm.qc.ca (I.D.); Racheal.Paul@ircm.qc.ca (R.P.) \\ 2 Département de Médecine, Université de Montréal, Montreal, QC H3T 1J4, Canada \\ 3 Department of Experimental Medicine, McGill University, Montreal, QC H4A 3J1, Canada \\ * Correspondence: Marie.Kmita@ircm.qc.ca; Tel.: +1-514-987-5749
}

Received: 13 October 2020; Accepted: 2 December 2020; Published: 7 December 2020

\begin{abstract}
Limb patterning relies in large part on the function of the Hox family of developmental genes. While the differential expression of Hox genes shifts from the anterior-posterior (A-P) to the proximal-distal (P-D) axis around embryonic day 11 (E11), whether this shift coincides with a more global change of A-P to $\mathrm{P}-\mathrm{D}$ patterning program remains unclear. By performing and analyzing the transcriptome of the developing limb bud from E10.5 to E12.5, at single-cell resolution, we have uncovered transcriptional trajectories that revealed a general switch from A-P to P-D genetic program between E10.5 and E11.5. Interestingly, all the transcriptional trajectories at E10.5 end with cells expressing either proximal or distal markers suggesting a progressive acquisition of P-D identity. Moreover, we identified three categories of genes expressed in the distal limb mesenchyme characterized by distinct temporal expression dynamics. Among these are Hoxa13 and Hoxd13 (Hox13 hereafter), which start to be expressed around E10.5, and importantly the binding of the HOX13 factors was observed within or in the neighborhood of several of the distal limb genes. Our data are consistent with previous evidence suggesting that the transition from the early/proximal to the late/distal transcriptome of the limb mesenchyme largely relies on HOX13 function. Based on these results and the evidence that HOX13 factors restrict Hoxa11 expression to the proximal limb, in progenitor cells of the zeugopod, we propose that HOX13 act as a key determinant of P-D patterning.
\end{abstract}

Keywords: limb patterning; single-cell RNA-seq; Hox genes

\section{Introduction}

Limb development in mouse initiates around E9.5, when the forelimb bud emerges from the lateral plate mesoderm. At this stage, undifferentiated mesenchymal cells receive signals from a group of ectodermal cells at the distal tip of the limb bud, referred to as the Apical Ectodermal Ridge (AER). The AER, located at the boundary between the dorsal and ventral ectoderm, expresses Fibroblast Growth Factors (Fgfs), notably $F g f 8$, whose function promotes limb bud growth and provides distal positional information to the underlying mesenchyme [1]. Concomitantly, Meis1/2 in the most proximal domain of the developing limb bud construes FGF signaling and contributes to establishing proximal positional information [2]. In parallel, cells at the distal-posterior margin of the limb bud express Sonic Hedgehog $(S h h)$, establishing a gradient of $\mathrm{SHH}$ signaling along the anterior-posterior (A-P) axis, which underlies the A-P patterning of the developing limb [3]. Genes whose transcriptional control is influenced by FGFs and SHH signaling include the Hox family of developmental genes, which contribute to the limb patterning along both the A-P and P-D axes (reviewed in, e.g., [4]). Hox genes belonging to the Hox A 
and HoxD clusters play a major role in limb development, as illustrated by the severe limb truncation resulting from the conditional inactivation of both gene clusters [5]. These genes are expressed in two phases. During the first phase, at early limb bud stages, Hox A/D genes are sequentially activated in time, from group 1, at one end of each cluster, to group 13 genes, located at the opposite end. During this first phase, Hox genes are expressed in a nested fashion along the A-P axis, with the late activated genes being posteriorly restricted [6]. The second phase of expression starts around E11.5, with HoxA/D genes from group 9 to 13 being differentially expressed along the P-D axis [4]. The transcriptional control of HoxA/D genes relies on remote cis-regulatory elements, located on both sides of each Hox cluster, forming the early/proximal and late/distal regulatory landscapes respectively [7,8]. Elegant studies have shown that the switch of activity from one landscape (downstream of the group 1 genes) to the other regulatory landscape (upstream of the group 13 genes) is associated with the differential expression of Hox genes along the P-D axis and thereby the establishment of the three limb segments along the P-D axis $[9,10]$. While there is a clear shift in Hox regulation around E11.5, whether it coincides with a more global change of A-P to $\mathrm{P}-\mathrm{D}$ patterning program remains unclear.

In this work, we have analyzed the complete transcriptome of the limb bud at three distinct stages, E10.5, E11.5 and E12.5, at single-cell resolution. Focusing on the limb bud mesenchyme, we have uncovered distinct transcriptional trajectories, which reveals a switch in the genetic program between E10.5 and E11.5. While at E10.5 three main transcriptional trajectories were found to coincide with distinct cell distribution along the A-P axis, at E11.5, the two main trajectories reflect differential P-D identity. Interestingly, the trajectories at E10.5 all end with cells expressing either proximal or distal markers suggesting a progressive acquisition of P-D identity. This finding is consistent with previous models suggesting that FGF signaling from the AER and proximal signaling from the flank create opposite signaling, which progressively results in the emergence of an intermediate identity separating proximal identity (stylopod) from distal identity (autopod) [1]. Our transcriptome analysis at E11.5 and E12.5 also identified three main categories of genes expressed in the distal limb mesenchyme characterized by distinct temporal expression dynamics. As anticipated from previous results, HOX13 binding was observed within or in the neighborhood of several of these genes, consistent with previous evidence suggesting that the transition from the early/proximal to the late/distal transcriptome of the limb mesenchyme largely relies on HOX13 function [11]. Finally, our data provide evidence that up to E12.5, the large majority of distal cells express the Hox13 genes while the other distal cells are cells in which Hox13 expression has been switched off and which undergo chondrogenic differentiation.

\section{Materials and Methods}

\subsection{Study Approval}

All studies with mice described in this article were approved by the Animal Care Committee of the Institut de Recherches Cliniques de Montréal (protocols \# 2015-14 and 2017-10).

\subsection{Mouse Lines}

The generation of Hoxa13:Cre and $m T / m G$ mouse lines was described elsewhere [12,13]. All mice were maintained in mixed background (C57BL/6X129). Noon of the day of the vaginal plug was considered as E0.5. Mice and embryos were genotyped by polymerase chain reaction (PCR) using genomic DNA extracted from tail biopsy specimens and yolk sacs, respectively.

\subsection{3'End Single-Cell RNA-seq}

Dissection of forelimb buds from Hoxa13:Cre/+;mT-mG embryos was performed at E10.5, E11.5 and E12.5. Forelimbs were dissected in cold 1XPBS, and after centrifugation at $300 \mathrm{rcf}$ for $5 \mathrm{~min}$ at $4{ }^{\circ} \mathrm{C}$, the forelimbs were incubated in dissociation buffer $(450 \mu \mathrm{L}$ of $0.25 \%$ Trypsin/EDTA (GIBCO), $50 \mu \mathrm{L} 10 \%$ bovine serum albumin (BSA), $1 \mu \mathrm{L}$ DNAseI (NEB)) for $10 \mathrm{~min}$ at $37^{\circ} \mathrm{C}$. After incubation, limb cells were 
gently mixed by pipetting up and down 10-15 times until they were dissociated, and then $10 \%$ final fetal bovine serum (FBS) was added. Dissociated cells were filtered using a cell strainer (40 $\mu \mathrm{m}$ Nylon, BD Falcon), and counted manually using a hemocytometer to determine the final volume in which to resuspend the cells and assessed for cell viability using $0.4 \%$ Trypan blue. After centrifugation at 300 rcf for $7 \mathrm{~min}$ at $4{ }^{\circ} \mathrm{C}$, the resulting pellet of cells was resuspended in the appropriate volume of 1XPBS, $0.04 \%$ BSA in order to achieve a concentration of 1500 cells $/ \mu \mathrm{L}$. Before being processed, dissociated cells were counted one more time and assessed for cell viability using $0.4 \%$ Trypan blue. The single-cell preparation was processed using Chromium Next GEM Chip G Single Cell Kit (10X Genomics PN 1000127) and Chromium Next GEM Single Cell 3' GEM, Library and Gel Bead Kit v3.1 (10X Genomics PN 1000128) following the manufacturer's recommendations. Briefly, forelimb cells were partitioned into gel beads in emulsion for cell lysis, barcoding with oligo-dT primers and reverse transcription in order to produce barcoded, full-length cDNA from poly-adenylated mRNA. cDNA library was amplified, fragmented and size selected. Samples were controlled at multiple steps during the procedure by running on BioAnalyzer. Libraries were sequenced on NovaSeq 6000 PE 26x98 (98-8-26).

\subsection{Single-Cell RNA-seq Data Analysis}

\subsubsection{Pre-Processing}

The Cell Ranger Single-Cell Software Suite version3.0.1 (10X Genomics ${ }^{\circledR}$ ) was used to perform sample demultiplexing, alignment, filtering, barcode counting, and UMI counting. Sequencing reads were aligned on a custom genome on which sequences of IRES-venus, tdTomato, Cre and eGFP were added to Mus_musculus.GRCm38.93. genome. At E10.5, 6402 cells were profiled, 6748 cells at E11.5 and 8592 cells at E12.5. Feature-barcode matrices were generated for each sample. Further analysis-including quality filtering, data normalization and scaling, identification of highly variable genes, dimensionality reduction, standard unsupervised clustering algorithms and the discovery of differentially expressed genes—was performed using the Seurat R package v3.1 [14,15].

\subsubsection{Quality Control and Filtering}

To exclude low-quality cells, we removed cells that had unique feature counts less than 1500 or over 7500. Our samples presented a number of unique transcripts per cell that were depending on the number of total reads detected per cell following a logarithmic function that can be presented as following: Unique Transcripts $=\alpha \log _{2.05}$ (\#total transcript) or (Unique Transcripts $)^{2.05}=b$ (\#total transcript). We considered cells presenting a beta inferior to 800 while having a total number of transcripts superior to 3000 as doublets and filtered them out. We also removed cells with more than $12.5 \%$ and less than $2 \%$ of the transcripts coming from mitochondrial genes. After quality filtering, the number of cells per sample was 6008 cells at E10.5, 5955 cells at E11.5 and 6972 cells at E12.5.

\subsubsection{Normalization and Scaling}

For the rest of the analysis, we analyzed each sample individually, but we also combined the three samples (E10.5, E11.5 and E12.5) using the merge function. The four Seurat objects were analyzed with the same parameters. We first normalized the data. Next, the data were scaled and during this step the mitochondrial contamination as well as cell cycle phases heterogeneity were 'regressed out' using the var.to.regress function in order to remove these sources of variation from our single-cell dataset. We removed cell cycle stages variation as we observed that without this step the influence of the cell cycle was taking over the cell identity variation during the clustering and was affected the downstream analysis to identify gene marker specific to a cell population. We used the CellCycleScoring function to assign cell cycle scores to each cell to do the scaling on this parameter. This scoring was based on the expression of cell cycle markers divided into G2/M phase markers, $S$ phase markers and cells that did not express any of these markers were identified as being in G1 phase. 


\subsubsection{Dimensional Reduction}

Using the FindVariableFeatures function, 3000 features were identified as highly variable from cell to cell. Next, the lowest (mean expression $<5$ th percentile) and the highest (mean expression $>80$ th percentile) expressed genes for each dataset were removed from the 3000 highly variable features list in order to improve the downstream analysis [16]. We then performed linear dimensional reduction using the Principal Component Analysis (PCA) method including 50 Principal Components (PCs) and to visualize the data we used the Uniform Approximation and Projection method (UMAP) [17].

\subsubsection{Clustering}

The clustering was performed using the Louvain algorithm [18,19] with a resolution of 0.3 . We used the subset function in order to create a new Seurat object containing only the mesenchymal cells clusters, identify previously, for E10.5, E11.5 and E12.5. Each new Seurat object was scaled, highly variable features were identified and linear (PCA) [20] and non-linear (UMAP) dimensional reductions. From these subsets, we used the WhichCells function of Seurat $[14,15]$ to select the Hoxa13 positive cells in the mesenchyme and we then used the function FindAllMarkers to identify the marker genes for the Hoxa13 positive mesenchymal cells and for the Hoxa13 negative mesenchymal cells. From the mesenchymal subsets at E10.5, E11.5 and E12.5 we used the WhichCells function to select the Hoxa13+;Hoxd13+;Gfp+ and the Hoxa13-;Hoxd13-;Gfp+ cells in the mesenchyme. We then created subsets, using the subset function, composed of the selected cells and we merged them to create a new Seurat object containing the Hoxa13+;Hoxd13+;Gfp + (Hox13+;Gfp + hereafter) cells and the Hoxa13-;Hoxd13-;Gfp+ (Hox13-;Gfp + hereafter) cells for each genotype. The new Seurat object was scaled, highly variable features were identified and linear (PCA) [20] and non-linear (UMAP) dimensional reductions were performed. We then used the function FindAllMarkers (min.pct $=0$ and logfc.threshold $=0.25$ ) to identify the marker genes for the Hox13+;Gfp+ and for the Hox13-;Gfp+ mesenchymal cells. We used DoHeatmap function to visualise the significant (p_val_adj <0.05) marker genes for each cell population. We used these marker genes to perform the gene ontology analysis using DAVID [21,22].

\subsubsection{Monocle3 Analysis}

We started the Monocle3 [23-25] analysis from the merged Seurat object (E10.5, E11.5 and E12.5), which was previously normalized, scaled with the removal of cell cycle and mitochondrial contamination variations, high variable features were identified with the removal of the highest and the lowest expressed genes and linear dimension was performed using PCA (see the Normalization and Scaling, and Dimensional Reduction sections). The function as.cell_data_set from the seurat-wrappers package was used to create the Monocle 3 cds object for further analysis. We performed non-linear (UMAP) dimensional reductions, we calculated the size factors to evaluate variation in the number of mRNAs per cell, we then clustered the cells using the Leiden algorithm [26] with a resolution of 0.0003 . Marker genes of each cluster were identified using the top_markers function. To analyze only the mesenchymal cells, we used the function choose_cells to subset the mesenchyme from E10.5, E11.5 and E12.5 samples, as well as E10.5 only and E11.5/E12.5 only. We clustered the subset objects using the Leiden algorithm and we used the function learn_graph to learn a principal graph in order to identify trajectories in function of the gene expression changes.

\subsection{Single-Cell ATAC-seq Data Analysis}

The single-cell ATAC-seq raw data were published [27] and can be found on GEO under the accession number GSE145657. Sequencing reads were aligned using Cell Ranger ATAC version1.1.0 $\left(10 \mathrm{X}\right.$ Genomics $\left.{ }^{\circledR}\right)$ and Signac version1.0.0 (extension of Seurat [14,15], developed by Tim Stuart and Avi Srivastava) was used to perform the analysis. We used the function cellranger-atac count from Cell Ranger ATAC version1.1.0 (10X Genomics $\left.{ }^{\circledR}\right)$ to perform read filtering and alignment on 
mm10, barcode counting, identification of transposase cut sites, detection of accessible chromatin peaks, cell calling and count matrix generation for peaks with the following parameters for the peak calling, window_size $=250$ and peak_merge_distance $=5$. We then used Signac version1.0.0 to analyze the Cell Ranger ATAC results. First, we created a Seurat object from the Cell Ranger ATAC files (filtered_peak_bc_matrix.h5 as the peak-feature-barcode matrices, singlecell.csv that contains per barcode information and fragments.tsv.gz that represents the list of unique fragments across all single cells). Next, we annotated using the UCSC style and we cleaned the data by eliminating cells which have a high ratio of mononucleosomal to nucleosome-free fragment sizes (i.e., >2.5), a low transcriptional start site (TSS) enrichment (i.e., <2), a very low and very high level of reads measured by the total number of fragments in peaks (i.e., $<2000$ and $>25,000$ ), a low fraction of fragments in peaks (i.e., <15) and reads overlapping with ENCODE Blacklist regions (i.e., >0.05). Normalization and linear dimensional reduction were performed using the latent semantic indexing (LSI) [28]. We then clustered the cells using the SLM algorithm at a resolution of 0.3 [29] and we visualized the data using UMAP [17] and the frequency of Tn5 integration as accessibility tracks.

\subsection{Whole-Mount In Situ Hybridization}

Whole-mount in situ hybridization was performed using standard procedure [27]. Briefly, embryos were rehydrated through a methanol series (100-30\%), washed in PBST (0.1\% Tween) and bleached for an hour on ice in the dark using 6\% hydrogen peroxide. Then, embryos were treated with Proteinase $\mathrm{K}$ at RT for $10 \mathrm{~min}$ for E10.5 embryos and for $15 \mathrm{~min}$ for E11.5 and E12.5 embryos. Following the Proteinase $\mathrm{K}$ treatment embryos were re-fixed with $4 \%$ paraformaldehyde (PFA). Next, embryos were hybridized with the Digoxigenin (DIG)-labelled riboprobes in hybridization buffer (5× SSC pH 4.5; 50\% deionized formamide; $1 \%$ SDS; $0.1 \%$ Tween; $5 \mathrm{mg} / \mathrm{mL}$ torula RNA, $0.5 \mathrm{mg} / \mathrm{mL}$ heparin) overnight at $68{ }^{\circ} \mathrm{C}$. Embryos were then washed with $1 \times$ TBS; $0.1 \%$ Tween, treated with $10 \%$ goat serum; 1\% BSA and incubated with alkaline phosphatase-conjugated anti-DIG antibodies (1/3000; Roche) overnight at $4{ }^{\circ} \mathrm{C}$. The coloration was achieved using nitrotetrazolium blue chloride (NBT)/ 5-bromo-4-chloro-3-indolyl-phosphate, 4-toluidine salt (BCIP) substrate (Roche). After staining, the samples were washed in PBS and post-fixed with 4\% PFA. Embryos were photographed using the Leica, Wetzlar, Germany M165FC stereomicroscope coupled to the DFC450.C camera. A minimum of three embryos per genotype was assayed for reproducibility $(n=3)$. Digoxigenin (DIG)-labelled antisense riboprobes were generated from cDNA using the following primers with the reverse primers containing the T7 promoter sequence: Efna1, forward: GTGACTGTCAATGGCAAAAT and reverse: TAATACGACTCACTATAGGGGAAATCTTGCAGAGATGCTG; Creb5, forward: CCACCCTCAGTCAGCTTACA and reverse: TAATACGACTCACTATAGGGATCATGAGCTTTC CCACCCA; Cpa2, forward: TGTGTTCTCCCAAACCTCCA and reverse: TAATACGACTCACTA TAGGGCACTGGCCTGGTAGATGACA; Cdh3, forward: TGCTGACTAGGGGGACAGTT and reverse: TAATACGACTCACTATAGGGCCCTCTCCATCCATGTCTGT. Hoxa13 and Bmp2 probes were previously described [30,31].

\subsection{Data Availability}

The scRNA-seq raw and processed data can be found on GEO under the accession number GSE158820. The scATAC-seq raw data were obtained from the accession numbers GSE145657. The ChIP-seq data for HOXA13, HOXD13 and H3K27ac are available on GEO under the accession number GSE81356. This study did not generate any unique code, and all analyses were performed in R using standard protocols from Seurat and Monocle3 [14,15,25]. All scripts associated with this manuscript are available upon request. 


\section{Results}

3.1. Transcriptional Trajectories in the Developing Forelimb Bud Reveal a Major Switch in the Developmental Program between E10.5 and E11.5

To gain a better understanding of the developmental program underlying limb patterning, we analyzed the transcriptome at single-cell resolution from mouse forelimb buds isolated from embryos at gestational day 10.5 (E10.5), E11.5 and E12.5 (Figure 1a). The dimensional reduction procedure applied to our single-cell RNA-seq (scRNA-seq) dataset was based on the identification of the 3000 most variable expressed genes, from which the lowest and highest expressed genes were removed. The resulting gene set was then used to conduct a Principal Component Analysis (PCA) for linear dimensional reduction (see Materials and Methods for details). To visualize the data, we then used the Uniform Approximation and Projection method (UMAP) that positions cells according to their transcriptome, with each dot representing a cell and neighboring dots corresponding to cells with the highest similarity of their transcriptome (Figure 1b). UMAP projection of our scRNA-seq dataset revealed that the majority of E10.5 cells are isolated from E11.5 and E12.5 cells (Figure 1b), suggesting a major transcriptional switch between E10.5 and E11.5. Cell clustering was then used to group cells according to similarities in gene expression, which showed a clear segregation between the main cell types, i.e., mesenchyme, ectoderm, myoblasts/myocytes, blood cells (Figures 1c,d and S1), validating the biological relevance of our dataset. The cell clustering revealed that the major transcriptional switch between E10.5 and E11.5 occurred within the mesenchymal cell population (Figures 1c,d and S1).

(a)
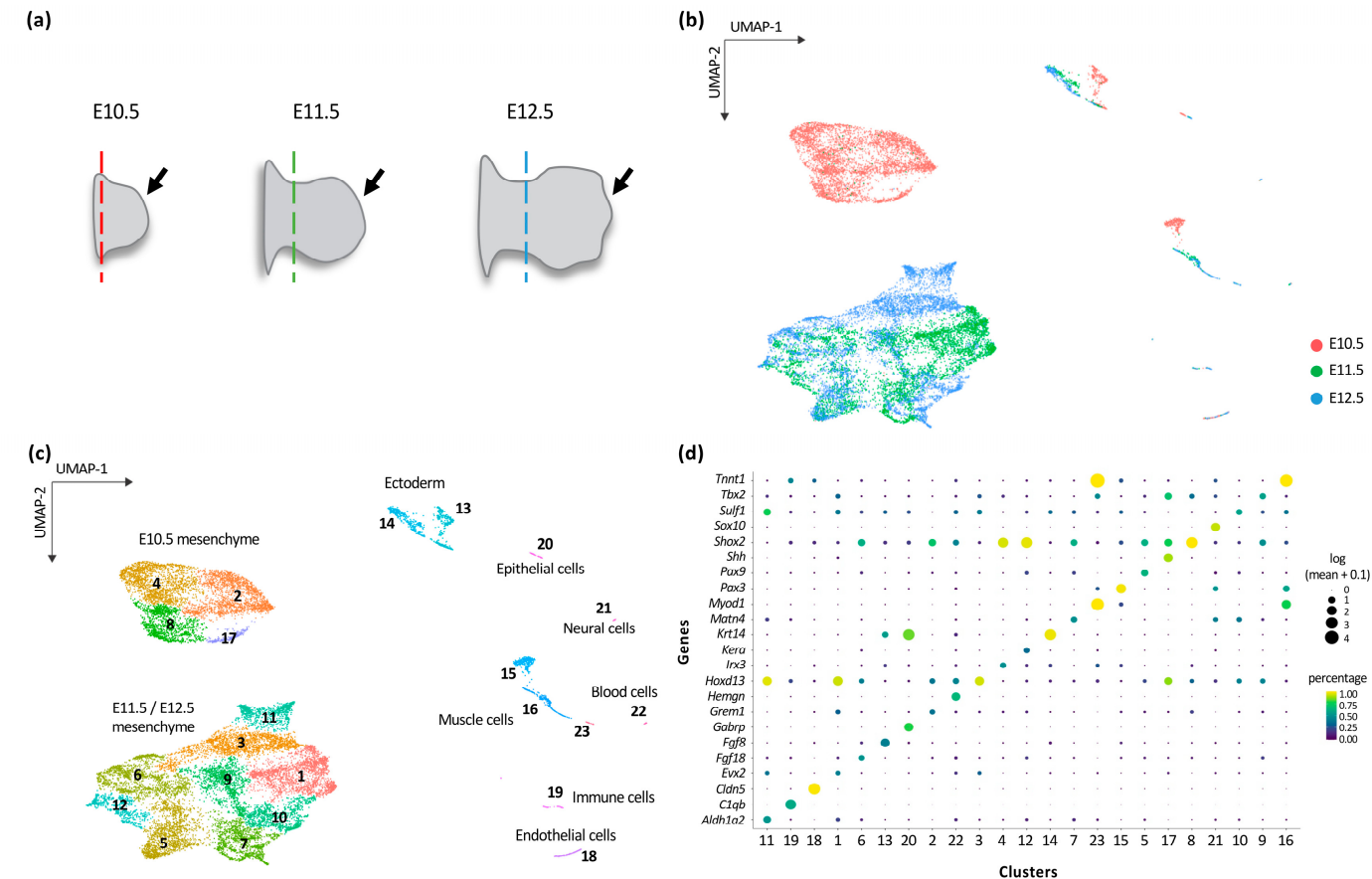

Figure 1. Identification of the different cell identity in the developing mouse limb bud. (a) Schematics illustrating mouse forelimb buds tissues used in this study: E10.5 (left, black arrow), E11.5 (middle, black arrow) and E12.5 (right, black arrow). The dashed color lines mark the position of the dissection. (b,c) UMAP visualization of 18,935 mouse forelimb buds cells, colored by developmental stages (6008 cells for E10.5 in red; 5955 cells for E11.5 in green and 6972 cells for E12,5 in blue) (b) and by cluster identity from Leiden clustering (c). The clusters were annotated based on the identification of marker genes. (d) Dot plot showing the expression of one selected marker gene per cluster. The size of the dot represents the mean of the expression level of the gene and its color represents the percentage of cells within the cluster in which that gene was detected. 
We thus focused subsequent analyses on the limb mesenchyme (Figure 2). A tentative identity for each cluster was established based on marker genes (Figures $1 \mathrm{~d}$ and $2 \mathrm{a}$ ). In order to determine gene expression changes between developmental programs, we first established trajectories using Monocle 3 workflow [23-25] (Figure 2a). A trajectory establishes relationships between cells according to their transcriptome such that the transcriptome of cells that belong to two distinct trajectories are more divergent than between cells that belong to the same trajectory. At E10.5, three discrete trajectories can be identified (Figure $2 b$ ). We first identified gene markers for each trajectory. The first trajectory is characterized by cells expressing Irx3, which marks the anterior cell population [32]. The second one is marked by Grem1 expression, which characterizes the central domain of the limb bud [33] and the third trajectory includes cells expressing Shh, located at the posterior margin of the developing limb bud [34]. These well-characterized markers allowed us to orient the UMAP projection and position the trajectories in the mouse limb bud (Figure $2 b$ ). We then used other well-known markers to validate the differential antero-posterior identity of the trajectories. Alx4, which is expressed in the anterior limb bud [35], was found in trajectory 1. Tbx2, expressed both in the anterior and posterior margins [36], was present in trajectories 1 and 3 and excluded from trajectory 2 while Hoxd12, expressed in the posterior half domain [37], was found in trajectories 2 and 3 and excluded from trajectory 1 . In contrast to genes having a differential antero-posterior expression, Meis1, which characterizes the proximal limb domain [38], was observed in the three trajectories. Of note, Hoxa13, which is expressed distally with a posterior bias $[39,40]$, was present at the extremity of trajectory 2 and 3 . Together, the distribution of these marker genes on the UMAP projection indicated that trajectory 1 corresponds to anterior limb cells, trajectory 2 to the central limb domain and trajectory 3 to the posterior limb (Figure 2b). These data thus suggested that antero-posterior patterning is the primary patterning event in early limb buds.

As far as the E11.5 and E12.5 cell population is concerned, we found two main trajectories, which split into 'secondary' branches. Of note, the extremity of almost all 'branches' is composed of E12.5 cells (Figure 2c). The marked segregation between E10.5 and E11.5 clustering prevented us from connecting the E10.5 trajectories with those of E11.5 and E12.5 limb bud cells. Nonetheless, based on the genes characterizing each trajectory, a clear switch could be observed whereby the main trajectories at E10.5 coincided with distinct A-P distribution while the two main trajectories at E11.5 and E12.5 limb buds correspond to a differential P-D identity as revealed by the distribution of cells expressing the proximal markers Shox 2 and Hoxa11 [41-43] and cells expressing the distal markers Hoxa13 and Hoxd13 [39,40](Figure 2c). 
(a)
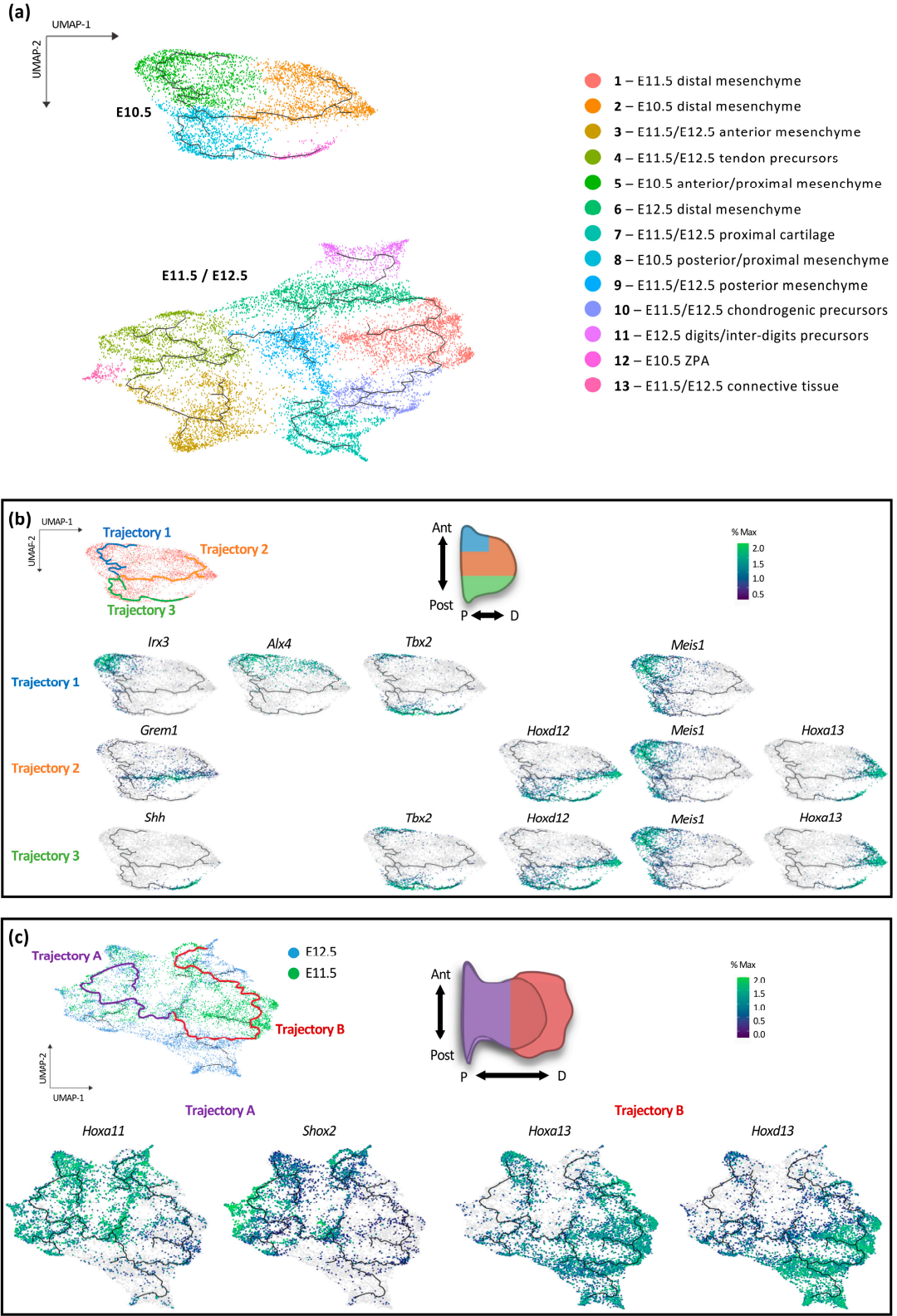

Figure 2. Characterization of the main transcriptional trajectories in the developing limb mesenchyme. (a) UMAP visualization of the forelimb bud mesenchymal cells colored according to cluster identity on which developmental trajectories, identified using Monocle 3, are represented. (b) Characterization of the three mesenchymal trajectories at E10.5, trajectory 1 is highlighted in blue, trajectory 2 in orange and trajectory 3 in green on the UMAP representation (top left). The E10.5 forelimb bud schematic indicates the orientation of the UMAP representation and the localization of the trajectories (middle top). Expression profiles of marker genes for each trajectory are shown below. Color scale indicated the scaled expression to percent of the maximum expression of all marker genes at E10.5 (top right). (c) Identification of the two main transcriptional trajectories at E11.5 (green cells) and E12.5 (blue cells), trajectory A is highlighted in purple and trajectory B in red (top left). E11.5 and E12.5 overlapping limb bud schematics indicate the orientation of the UMAP representation and the localization of the trajectories (middle top). Expression profiles of marker genes for each trajectory are shown below (bottom left and right, respectively). Color scale indicated the scaled expression to percent of the maximum expression of all marker genes at E11.5 and E12.5 (top right). Ant: anterior, Post: posterior, P: proximal, D: distal. 


\subsection{Individual Transcriptional Trajectories Are Characterized by a Specific Hox Code}

We next examined the distribution of Hox-expressing cells to further analyze the trajectories in the context of limb bud patterning into three distinct domains along the P-D axis-the stylopod, zeugopod and autopod. We chose the Hox genes for their well-characterized differential expression along both the P-D and A-P axes and their known impact on the limb architecture (reviewed in, e.g., [4]). At E10.5, cells expressing Hoxa9, $d 9, a 10$ and d10, which pattern the stylopod, are present in all three trajectories (Figure 3a). Hoxa11 and Hoxd11, responsible for the zeugopod formation, are expressed in cells belonging to trajectories 2 and 3, which also express Hoxd12 but to a lesser extent. Finally, cells expressing Hoxd13 are observed in trajectory 3 and the extremity of trajectory 2 while cells expressing Hoxa13 are only found at the extremity of trajectories 2 and 3 (Figure 3a). Together, these data revealed that each trajectory is characterized by the expression of a specific combination of Hox genes, which in turn further support the differential A-P identity of the three transcriptional trajectories identified at E10.5. For limb tissue at E11.5 and 12.5, the dissection plan did not include the most proximal cells to avoid 'contamination' from flank tissue (Figure 1a). As a result, rare Meis1/2-expressing cells were observed in the E11.5 and E12.5 samples (Figure S2). This together with the relative prevalence of the distal limb tissue as compared to the proximal tissue explains the large proportion of E11.5 and E12.5 mesenchymal cells expressing Hoxa13 and Hoxd13 (Figure S2). We also found that Hoxa9, Hoxa10, Hoxd10 and Hoxd11 are expressed in virtually all limb bud cells (Figure 3b). Hoxa11 and Hoxa13 have an almost mutually exclusive distribution (Figure 3c), as expected from previous whole mount in situ hybridization data [43-45] and consistent with the HOX13-mediated repression of Hoxa11 [43]. Of note, there are few cells that express both Hoxa11 and Hoxa13, which possibly correlate with cells in which Hoxa13 is transcribed but the HOXA13 protein is not yet produced/functional (Figure 3c, red arrows). These cells also express the proximal marker Shox2 (Figure 3c, red arrows), suggesting that these cells may not have yet acquired a definitive P-D identity. We also observed that Hoxd9 is expressed in all limb bud cells at E10.5 but is barely expressed in distal cells at E11.5 and E12.5, similar to Hoxa11 (Figure 3c, purple arrows). Together, these data highlight the fact that, at E11.5-E12.5, only five of the Hox genes have a spatially restricted expression, namely Hoxd9, Hoxa11, Hoxd12, Hoxa13 and Hoxd13 (Figure 3c), while the others are expressed in virtually all cells though with distinct expression levels along the P-D axis (Figure 3b).

\subsection{Dynamic Expression of Distal Genes Largely Relies on HOX13 Function}

We next focused our analysis on distal mesenchymal cells. We used Hoxa13 (Figure 4a), which is expressed in the progenitor cells of the hand and wrist, as a marker to identify distal mesenchymal cells [12]. Analysis of the transcriptome of distal cells at E10.5, E11.5 and E12.5 revealed three categories of 'distal' genes based on the temporal dynamics of their expression (Figures $4 \mathrm{~b}$ and S3). Category 1 corresponds to genes not expressed at E10.5 but becoming expressed in Hoxa13-positive cells at E11.5 (e.g., Creb5, Bmp2, Efna1, and Stmn2). Category 2 represents genes whose transcription is found among the distal cells at E10.5 and E11.5 while only rare cells at E12.5 express these genes (e.g., Gja3, Cpa2, and $C b f a 2 t 3)$. Finally, Category 3 genes are found expressed over the three stages in a subset of distal cells (e.g., Cdh3, Lmo2, Tfap2b, Jag1, and Hey1).

To gain insights as to whether these genes are regulated by HOX13 TFs, we checked whether HOXA13 and/or HOXD13 proteins bind in their vicinity and analyzed chromatin accessibility at single-cell resolution (scATAC-seq). HOXA13, HOXD13 and H3K27ac ChIP-seq data as well as the scATAC-seq data were those previously generated from E11.5 limb buds [11,27]. Analysis of scATAC-seq and ChIP-seq data reveals that genes from all three categories are in an accessible state and decorated with the H3K27ac active mark in the entire limb bud (Figures 5a and S4), suggesting that their distal expression is due to transcriptional enhancers specifically active in the distal mesenchyme. Interestingly, we found that genes from all three categories are associated with HOX13 ChIP-seq peaks, either within the gene (e.g., Creb5), downstream (e.g., Gja3 and Efna1), or upstream (e.g., Bmp2 and Tfap2b). Loci bound by HOX13 are characterized by a cell-specific accessibility (Figure 5b). Notably, the HOX13-bound loci 
are all in an inaccessible/reduced accessibility in the 'proximal' and 'middle 1' clusters, while they are all in an accessible state in the 'distal' clusters. Together with the evidence that these loci are decorated with the active H3K27ac histone mark (Figure 5b), these data suggest that the HOX13-bound loci correspond to distal-specific regulatory elements. The HOX13-bound loci are also in an accessible state in the cluster referred to as 'middle 2', which may coincide with cells expressing both Hox13 and proximal markers, as described above, which possibly corresponds to cells that have not yet acquired their definitive P-D identity. Although our data do not demonstrate whether the HOX13-bound loci reported here are the bona fide regulatory elements controlling these distal genes, the evidence that these loci are in an accessible state and coincide with regions decorated by the active H3K27ac histone mark (Figure 5b) supports a direct contribution of HOX13 in the regulation of this set of distal genes.

(a)

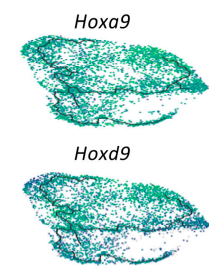

(b)

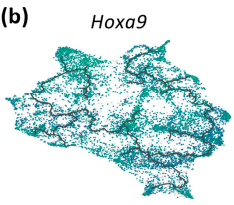

Hoxa10

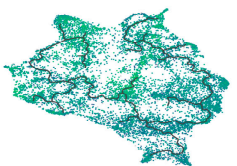

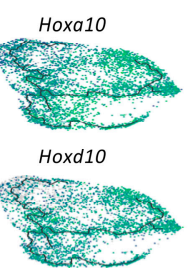

Hoxd11

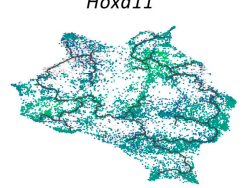

Hoxd 10

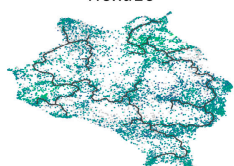

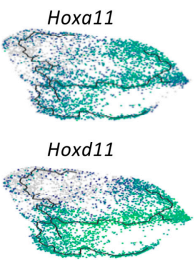

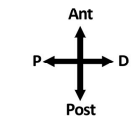

Hoxd12

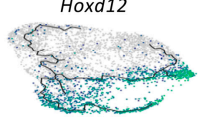

Hoxd9
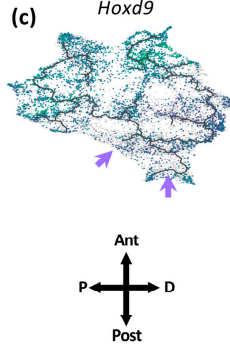

Hoxa11

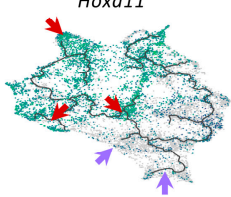

Hoxd12
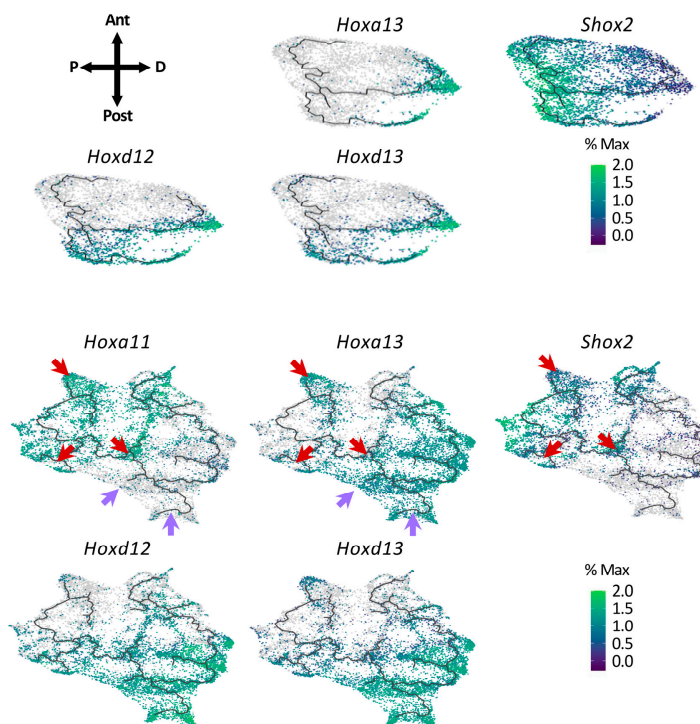

Hoxa13

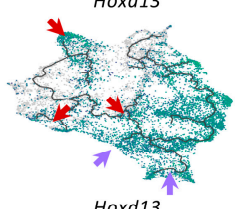

Hoxd13

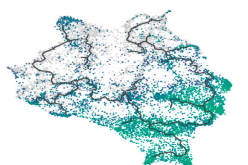

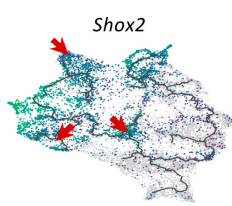

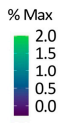

Figure 3. Distribution of Hox-expressing cells along the transcriptional trajectories during limb bud development. (a) UMAP visualization of the mesenchymal cells expressing the most 5' Hoxa and Hoxd genes (Hoxa9, Hoxd9, Hoxa10, Hoxd10, Hoxa11, Hoxd11, Hoxd12, Hoxa13 and Hoxd13) as well as the cells expressing Shox 2 at E10.5. Color scale indicated the scaled expression to percent of the maximum expression of all genes at E10.5. Orientation of the UMAP representation is indicated by the cross arrow schematic. (b,c) UMAP visualization of E11.5 and E12.5 mesenchymal cells expressing Hoxa9, Hoxa10, Hoxd10 and Hoxd11 in the all mesenchyme (b) and expressing Hoxd9, Hoxa11, Hoxd12, Hoxa13, Hoxd13 and Shox 2 in restricted areas of the mesenchyme (c). Red arrows indicate co-expression areas of Hoxa11, Hoxa13 and Shox2, and light purple arrows show the areas in which Hoxd9 and Hoxa11 are not expressed but Hoxa13 is expressed (c). Color scale indicated the scaled expression to percent of the maximum expression of all genes at E11.5 and E12.5. Orientation of the UMAP representation is indicated by the cross arrow schematic. Ant: anterior, Post: posterior, P: proximal, and D: distal. 
E10.5

(a)

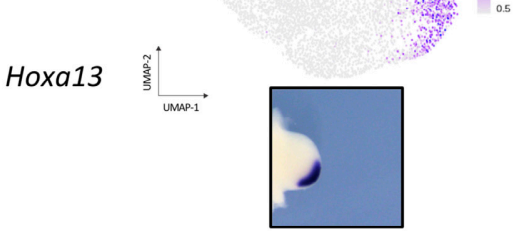

(b)
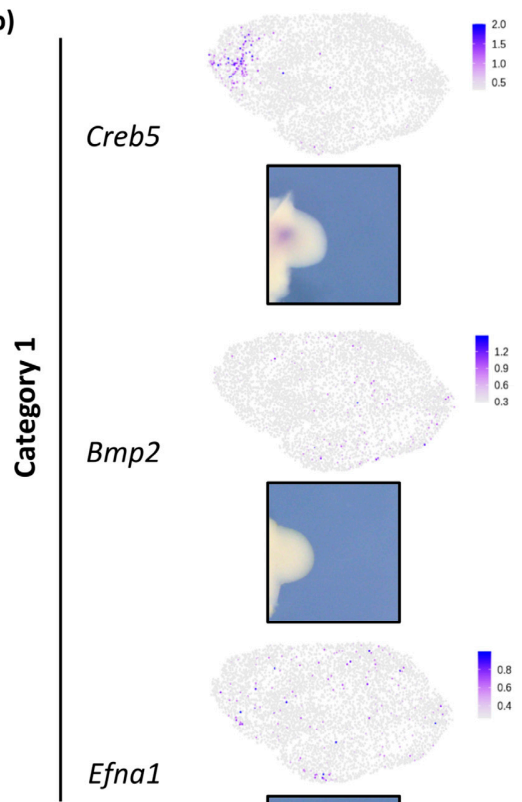

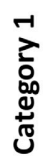

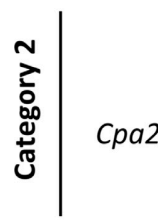
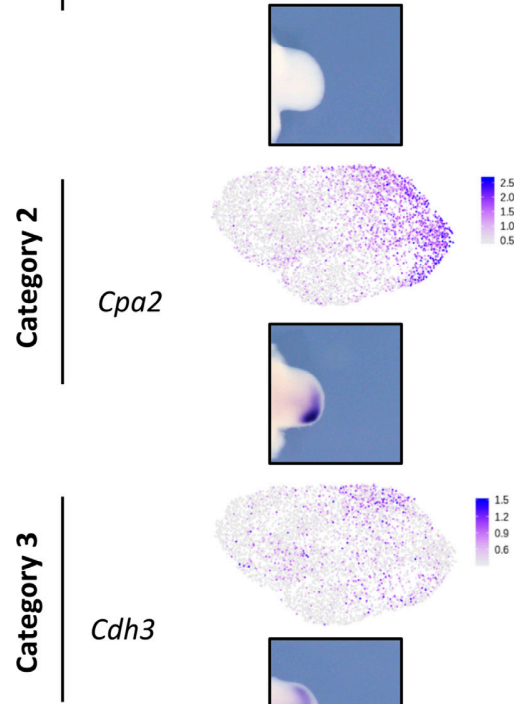

E11.5

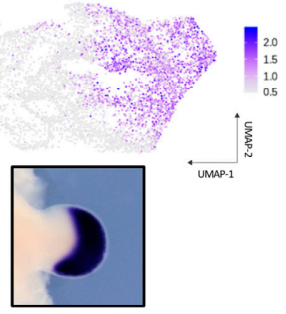

I:
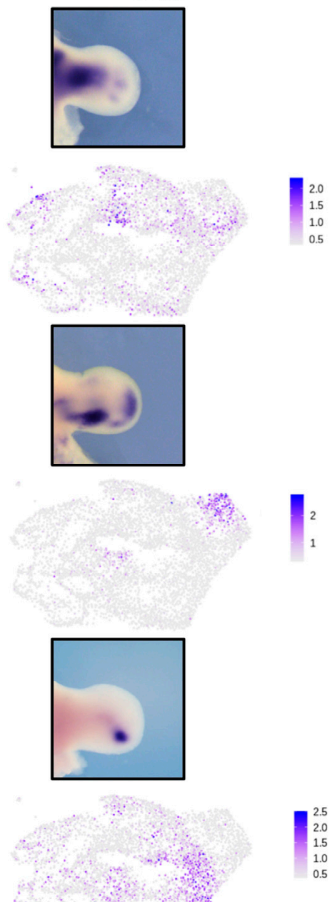

$\boldsymbol{W}_{20}^{25}$
1.5
10
10.5
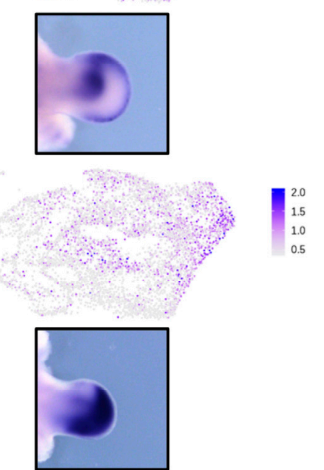
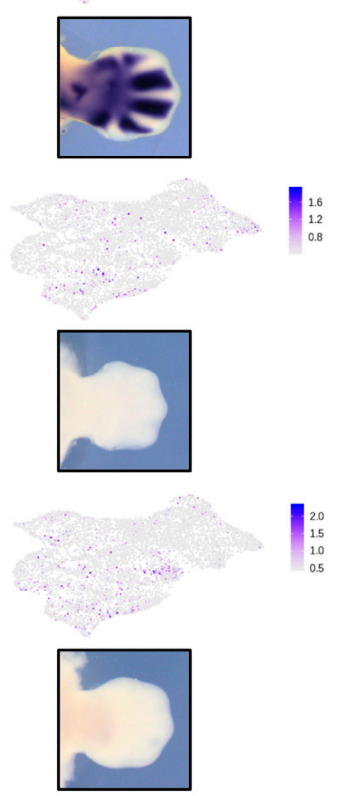

E12.5
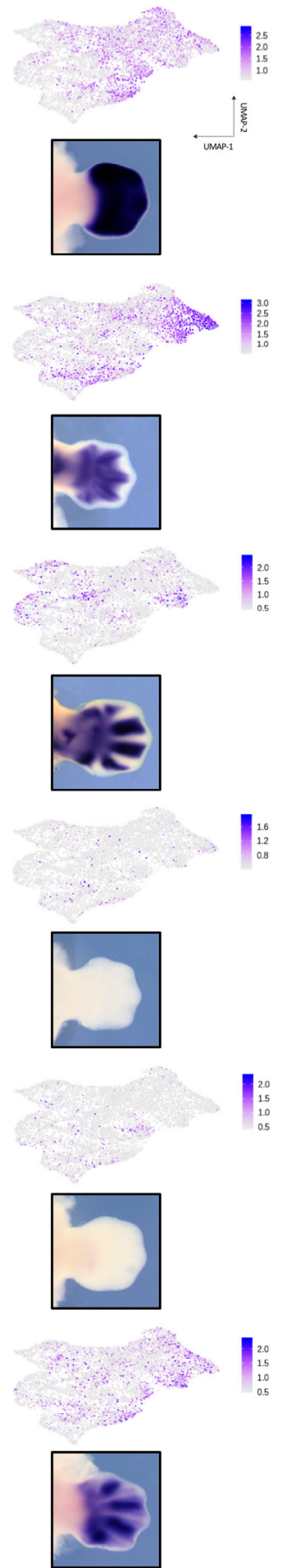

Figure 4. Dynamic expression of 'distal' genes during limb bud development. (a) UMAP visualization of the mesenchymal cells expressing Hoxa13 and whole-mount in situ hybridization for Hoxa13 in WT mouse forelimb bud at E10.5, E11.5 and E12.5. (b) UMAP visualization and whole-mount in situ hybridization of the distal genes Creb5, Bmp2, Efna1 belonging to Category 1, Cpa2 belonging to category2 and Cdh3 belonging to Category 3, in E10.5, E11.5 and E12.5 WT mouse forelimb buds. Color scale represents the scaled expression for each gene. 
(a)

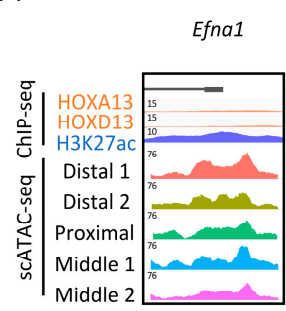

(b)

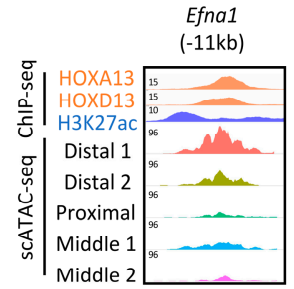

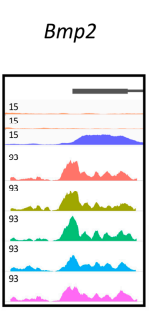

Promoters of the distal genes
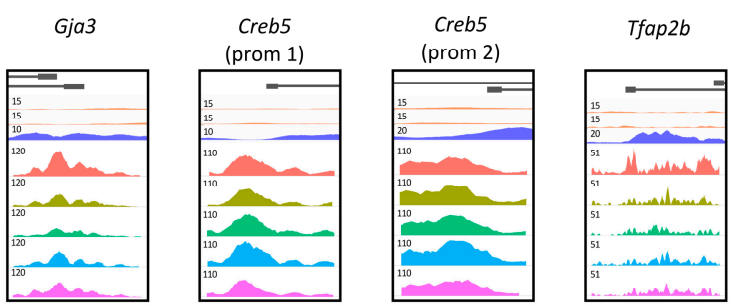

Loci bound by HOX13 at the distal genes
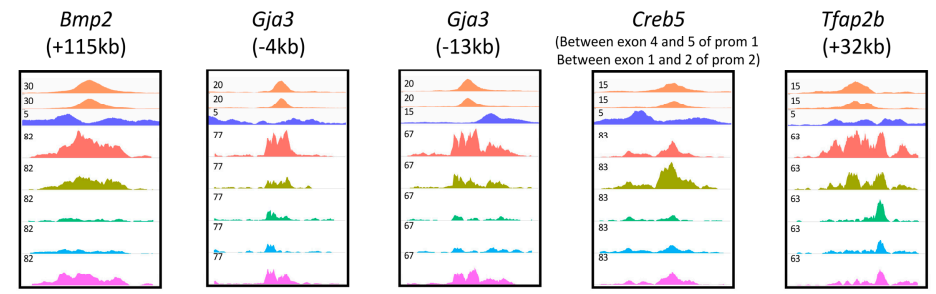

Figure 5. HOX13 binding at regulatory loci of distal genes correlates with distal-specific chromatin opening. $(\mathbf{a}, \mathbf{b})$ Genome views of HOXA13, HOXD13 and H3K27ac ChIP-seq (IGV) as well as scATAC-seq signals (Signac) at distal genes promoters (a) and at their previously identified or potential distal limb enhancers (b) in E11.5 mouse forelimb buds. Prom: promoter.

While Hoxa13 and Hoxd13 establish the distal identity in the developing limb bud, how the naturally occurring termination of their expression impacts the fate of distal limb cells remains elusive. To address this question, our scRNA-seq analysis was performed using limb buds from the Hoxa13Cre/+; $m \mathrm{~T} / m \mathrm{~m}$ line, which allows the identification of cells originating from Hoxa13-expressing cells, including those that do not express Hoxa13 anymore (Hoxa13-;Gfp+ cell population in our scRNA-seq data). Previous analysis of the Hoxa13Cre/+; $m$ T/mG mice provided evidence that, in the forelimb mesenchyme, all Hoxa13-expressing cells are progenitors of the hand and wrist [12], i.e., all Gfp-expressing mesenchymal cells in Hoxa13Cre/+; $m T / m G$ limb buds are cells with distal identity. We thus compared the transcriptome of Gfp-expressing cells which express neither Hoxa13 nor Hoxd13 (Hox13-;Gfp+) and those expressing Gfp, Hoxa13 and Hoxd13 (Hox13+; Gfp+). At E10.5, we identified only five Hox13-;Gfp + cells while 108 and 309 Hox13-;Gfp + cells were found at E11.5 and E12.5 respectively (Figure S5a). We thus focused our analysis on the E11.5 and E12.5 datasets. Transcriptome comparison between Hox13-;Gfp+ and Hox13+;Gfp+ cells revealed that the termination of Hoxa13 and Hoxd13 expression in distal cells is primarily associated with the upregulation of chondrogenic markers (Figure S5b,c). This indicated that the distal limb cells in which Hoxa13 and Hoxd13 expression is switched off are, at least up to E12.5, distal cells undergoing chondrogenic differentiation.

\section{Discussion}

In an attempt to gain further insights into the developmental program underlying limb patterning, we have performed and analyzed scRNA-seq experiments using isolated mouse forelimb buds from three stages, namely E10.5, E11.5 and E12.5. Analysis of this scRNA-seq dataset revealed a number of features characterizing the limb developmental program, including features previously reported $[1,9,11]$ which validate our experimental approach.

Our analysis highlights a major transition in gene expression between E10.5 and E11.5 and suggests a switch from a prevalent A-P patterning program in the early limb bud to a prevalent $\mathrm{P}-\mathrm{D}$ program at E11.5-E12.5. Indeed, while the main transcriptional trajectories at E10.5 are associated with distinct A-P markers, at E11.5-E12.5 there is a switch from three to two main trajectories, which are characterized by the expression of respectively proximal and distal markers. Next, we have investigated the distribution 
of cells expressing Hox genes, which are known for their critical function in establishing the architecture of the limb skeleton (e.g., [4]). At E10.5, the distribution of the Hox transcripts varies between the three identified trajectories according to their differential A-P expression, further supporting that at E10.5, each trajectory corresponds to a distinct limb bud domain along the A-P axis. At E11.5 and E12.5, based on our scRNA-seq dataset, Hox genes expressed in limb buds can be categorized into two groups: those expressed in virtually all cells, though with distinct expression levels (Hoxa9, a10, $d 10$ and 111 ) and those only expressed in a subset of limb cells (Hoxd9, Hoxa11, Hoxd12, Hoxd13 and Hoxa13). Previous work provided evidence that the proximal restriction of Hoxa11 expression is the consequence of HOX13-mediated regulation, whereby HOX13 triggers antisense transcription at the Hoxa11 locus, which, as a result, prevents Hoxa11 expression in the distal limb bud [43]. In this view, the key event that underlies Hox differential expression along the P-D axis of the developing limb, at least the qualitative aspect of it, is the regulatory mechanism responsible for Hox 13 transcriptional activation being restricted to the distal domain of the developing limb bud. This model is consistent with the evidence that the Hox13 function is required for the silencing of the regulatory landscape responsible for the proximal limb expression of the HoxD genes $[10,11]$. Interestingly, we found that a subset of cells expresses both Hox13 and Hoxa11, raising the possibility that these cells coincide with cells in which the Hox13 genes are expressed but the HOX13 proteins are either not yet produced or not yet functional. These cells also express the proximal maker Shox 2 suggesting that they have not yet acquired a definitive P-D identity. These cells could correspond to the progenitors of the wrist, located in between the zeugopod and the digits. This latter view is consistent with previous evidence showing that Hoxa13-expressing cells are progenitor cells of both the digits and wrist [12].

Based on the importance of Hox 13 distal-specific expression in establishing distal limb identity, we have further focused the analysis of our scRNA-seq dataset on genes expressed in Hox13-expressing cells. We found three categories of genes based on the developmental dynamics of their expression. Analysis of randomly selected genes from each category revealed HOX13 binding within the genes or within a $100 \mathrm{~kb}$ window around the genes. Based on the evidence that these HOX13-bound loci are in an accessible state specifically in distal limb cells and are marked by H3K27ac, which reflects a transcriptionally active status, it is likely that the majority of distally expressed genes are direct targets of HOX13. Accordingly, previous work provided evidence that the activity of distal-specific regulatory modules largely relies on HOX13 function [11,27]. These results, together with the evidence that an overall transition from A-P to P-D patterning occurs concomitantly with the beginning of Hox 13 expression, support a model whereby the HOX13 transcription factors have a prevalent role in the P-D patterning process.

Among the three categories of genes expressed in the distal limb bud, Category 1 are genes that become expressed at E11.5 and include genes whose activation in distal cells relies on HOX13 pioneer activity, as exemplified with Bmp2 [27]. Category 2 and 3 genes are those already expressed at E10.5 and thereby include upstream regulator(s) of the Hox13 genes as well as genes regulated by HOX13. Category 2 genes differ from Category 3 by being downregulated at E12.5, suggesting that HOX13 are not the primary factors controlling the expression of Category 2 genes, at least after E11.5. This category includes Gja3, known to be expressed in human chondrocytes [46] and Cpa2 [25]. Finally, Category 3 represents genes expressed at the three stages examined and include Tfap $2 b$, whose function is associated with digit formation [47].

The last aspect that we investigated in this study was to establish which cell fate(s) is associated with the termination of Hox13 expression. Taking advantage of a genetic lineage tracing tool, we were able to analyze the transcriptome of distal cells in which Hoxa13 and Hoxd13 are not expressed anymore. At least up to E12.5, these cells represent a small fraction of distal cells and are cells undergoing chondrogenic differentiation. However, it remains to be established whether chondrogenic differentiation can only be implemented upon the termination of Hoxa13 and Hoxd13 expression or is part of the mechanism that switch-off both genes. 


\section{Conclusions}

In summary, the present analysis of scRNA-seq data from mouse forelimb buds from E10.5 to E12.5 highlights a major transcriptional transition between E10.5 and E11.5, with transcriptional trajectories switching from A-P to P-D patterning programs. This study also identifies a subpopulation of limb mesenchymal cells, which have either not yet resolved their P-D identity at E12.5 or have an intermediate identity. These cells could correspond to progenitor cells of the wrist, which start forming at a stage when digit and zeugopod anlagen are clearly distinguishable. Finally, our scRNA-seq data identify three categories of genes expressed in Hox13-expressing cells, all three including genes whose transcriptional control involves HOX13 function. This later dataset should be useful for future studies aimed at unraveling the genetic network, triggering the emergence of digits as well as identifying transcription factors responsible for the distal restriction of the HOX13 pioneer factors.

Supplementary Materials: The following are available online at http://www.mdpi.com/2221-3759/8/4/31/s1, Figure S1: Expression profiles of the different gene markers in the developing mouse limb bud, Figure S2: Prevalence of the distal limb tissue as compared to proximal tissue, Figure S3: Examples of dynamic expression of distal genes during limb bud development, Figure S4: Annotation of the clusters identified by scATAC-seq and Figure S5: Analysis of the Hox13-;Gfp + cell population in the developing limb mesenchyme.

Author Contributions: Conceptualization, I.D. and M.K.; methodology, I.D.; validation, I.D. and R.P.; formal analysis, I.D.; investigation, I.D. and R.P.; resources, M.K.; data curation, I.D.; writing-original draft preparation, I.D. and M.K.; writing-review and editing, I.D., R.P. and M.K.; visualization, I.D., R.P. and M.K.; supervision, M.K.; project administration, M.K.; funding acquisition, M.K. All authors have read and agreed to the published version of the manuscript.

Funding: This research was funded by the Canadian Institutes of Health Research, grant number PJT-162143. I.D was supported by the IRCM-Jean Coutu fellowship.

Acknowledgments: We are grateful to lab members for insightful discussions and sharing reagents, Jessica Barthe for mouse management, Odile Neyret for NGS libraries preparation, Yasser Bouklouch, Alexandre Mayran, Virginie Calderon and Caroline Grou for their help in NGS analysis. Bioinformatics analyses were supported by Calcul Quebec (www.calculquebec.ca) and Compute Canada (www.computecanada.ca).

Conflicts of Interest: The authors declare no conflict of interest.

\section{References}

1. Lewandoski, M.; Sun, X.; Martin, G.R. Fgf8 signalling from the AER is essential for normal limb development. Nat. Genet. 2000, 26, 460-463. [CrossRef] [PubMed]

2. Delgado, I.; López-Delgado, A.C.; Roselló-Díez, A.; Giovinazzo, G.; Cadenas, V.; Fernández-de-Manuel, L.; Sánchez-Cabo, F.; Anderson, M.J.; Lewandoski, M.; Torres, M. Proximo-distal positional information encoded by an Fgf-regulated gradient of homeodomain transcription factors in the vertebrate limb. Sci. Adv. 2020, 6, eaaz0742. [CrossRef]

3. Panman, L.; Zeller, R. Patterning the limb before and after SHH signalling. J. Anat. 2003, 202, 3-12. [CrossRef] [PubMed]

4. Zakany, J.; Duboule, D. The role of Hox genes during vertebrate limb development. Curr. Opin. Genet. Dev. 2007, 17, 359-366. [CrossRef] [PubMed]

5. Kmita, M.; Tarchini, B.; Zàkàny, J.; Logan, M.; Tabin, C.J.; Duboule, D. Early developmental arrest of mammalian limbs lacking HoxA/HoxD gene function. Nature 2005, 435, 1113-1116. [CrossRef]

6. Tarchini, B.; Duboule, D. Control of Hoxd genes' collinearity during early limb development. Dev. Cell 2006, 10, 93-103. [CrossRef] [PubMed]

7. Berlivet, S.; Paquette, D.; Dumouchel, A.; Langlais, D.; Dostie, J.; Kmita, M. Clustering of Tissue-Specific Sub-TADs Accompanies the Regulation of HoxA Genes in Developing Limbs. PLoS Genet. 2013, 9, e1004018. [CrossRef] [PubMed]

8. Lonfat, N.; Montavon, T.; Darbellay, F.; Gitto, S.; Duboule, D. Convergent evolution of complex regulatory landscapes and pleiotropy at Hox loci. Science 2014, 346, 1004-1006. [CrossRef]

9. Andrey, G.; Montavon, T.; Mascrez, B.; Gonzalez, F.; Noordermeer, D.; Leleu, M.; Trono, D.; Spitz, F.; Duboule, D. A switch between topological domains underlies HoxD genes collinearity in mouse limbs. Science 2013, 340, 1234167. [CrossRef] 
10. Beccari, L.; Yakushiji-Kaminatsui, N.; Woltering, J.M.; Necsulea, A.; Lonfat, N.; Rodríguez-Carballo, E.; Mascrez, B.; Yamamoto, S.; Kuroiwa, A.; Duboule, D. A role for HOX13 proteins in the regulatory switch between TADs at the HoxD locus. Genes Dev. 2016, 30, 1172-1186. [CrossRef]

11. Sheth, R.; Barozzi, I.; Langlais, D.; Osterwalder, M.; Nemec, S.; Carlson, H.L.; Stadler, H.S.; Visel, A.; Drouin, J.; Kmita, M. Distal Limb Patterning Requires Modulation of cis-Regulatory Activities by HOX13. Cell Rep. 2016, 17, 2913-2926. [CrossRef] [PubMed]

12. Scotti, M.; Kherdjemil, Y.; Roux, M.; Kmita, M. A Hoxa13:Cre mouse strain for conditional gene manipulation in developing limb, hindgut, and urogenital system. Genesis 2015, 53, 366-376. [CrossRef] [PubMed]

13. Muzumdar, M.D.; Tasic, B.; Miyamichi, K.; Li, L.; Luo, L. A global double-fluorescent Cre reporter mouse. Genesis 2007, 45, 593-605. [CrossRef] [PubMed]

14. Butler, A.; Hoffman, P.; Smibert, P.; Papalexi, E.; Satija, R. Integrating single-cell transcriptomic data across different conditions, technologies, and species. Nat. Biotech. 2018, 36, 411-420. [CrossRef]

15. Stuart, T.; Butler, A.; Hoffman, P.; Hafemeister, C.; Papalexi, E.; Mauck, W.M., III; Hao, Y.; Stoeckius, M.; Smibert, P.; Satija, R. Comprehensive Integration of Single-Cell Data. Cell 2019, 177, 1888-1902.e21. [CrossRef]

16. Aztekin, C.; Hiscock, T.W.; Marioni, J.C.; Gurdon, J.B.; Simons, B.D.; Jullien, J. Identification of a regeneration-organizing cell in the Xenopus tail. Science 2019, 364, 653. [CrossRef]

17. McInnes, L.; Healy, J.; Melville, J. UMAP: Uniform Manifold Approximation and Projection for Dimension Reduction. arXiv 2020, arXiv:1802.03426. Available online: https://arxiv.org/abs/1802.03426 (accessed on 13 October 2020).

18. Blondel, V.D.; Guillaume, J.-L.; Lambiotte, R.; Lefebvre, E. Fast unfolding of communities in large networks. J. Stat. Mech. Theory Exp. 2008, 2008, P10008. [CrossRef]

19. Lancichinetti, A.; Fortunato, S. Community detection algorithms: A comparative analysis. Phys. Rev. E 2009, 80, 056117. [CrossRef]

20. Jolliffe, I.T.; Cadima, J. Principal component analysis: A review and recent developments. Phil. Trans. R. Soc. A 2016, 374, 20150202. [CrossRef]

21. Huang, D.W.; Sherman, B.T.; Lempicki, R.A. Bioinformatics enrichment tools: Paths toward the comprehensive functional analysis of large gene lists. Nucleic Acids Res. 2009, 37, 1-13. [CrossRef] [PubMed]

22. Huang, D.W.; Sherman, B.T.; Lempicki, R.A. Systematic and integrative analysis of large gene lists using DAVID bioinformatics resources. Nat. Prot. 2009, 4, 44-57. [CrossRef] [PubMed]

23. Trapnell, C.; Cacchiarelli, D.; Grimsby, J.; Pokharel, P.; Li, S.; Morse, M.; Lennon, N.J.; Livak, K.J.; Mikkelsen, T.S.; Rinn, J.L. The dynamics and regulators of cell fate decisions are revealed by pseudotemporal ordering of single cells. Nat. Biotech. 2014, 32, 381-386. [CrossRef] [PubMed]

24. Qiu, X.; Mao, Q.; Tang, Y.; Wang, L.; Chawla, R.; Pliner, H.A.; Trapnell, C. Reversed graph embedding resolves complex single-cell trajectories. Nat. Methods 2017, 14, 979-982. [CrossRef] [PubMed]

25. Cao, J.; Spielmann, M.; Qiu, X.; Huang, X.; Ibrahim, D.M.; Hill, A.J.; Zhang, F.; Mundlos, S.; Christiansen, L.; Steemers, F.J.; et al. The single-cell transcriptional landscape of mammalian organogenesis. Nature 2019, 566, 496-502. [CrossRef] [PubMed]

26. Traag, V.A.; Waltman, L.; van Eck, N.J. From Louvain to Leiden: Guaranteeing well-connected communities. Sci. Rep. 2019, 9, 5233. [CrossRef]

27. Desanlis, I.; Kherdjemil, Y.; Mayran, A.; Bouklouch, Y.; Gentile, C.; Sheth, R.; Zeller, R.; Drouin, J.; Kmita, M. HOX13-dependent chromatin accessibility underlies the transition towards the digit development program. Nat. Commun. 2020, 11, 2491. [CrossRef]

28. Cusanovich, D.A.; Daza, R.; Adey, A.; Pliner, H.A.; Christiansen, L.; Gunderson, K.L.; Steemers, F.J.; Trapnell, C.; Shendure, J. Multiplex single-cell profiling of chromatin accessibility by combinatorial cellular indexing. Science 2015, 348, 910. [CrossRef]

29. Waltman, L.; van Eck, N.J. A smart local moving algorithm for large-scale modularity-based community detection. Eur. Phys. J. B 2013, 86, 471. [CrossRef]

30. Warot, X.; Fromental-Ramain, C.; Fraulob, V.; Chambon, P.; Dollé, P. Gene dosage-dependent effects of the Hoxa-13 and Hoxd-13 mutations on morphogenesis of the terminal parts of the digestive and urogenital tracts. Development 1997, 124, 4781-4791.

31. Dickinson, M.E.; Kobrin, M.S.; Silan, C.M.; Kingsley, D.M.; Justice, M.J.; Miller, D.A.; Ceci, J.D.; Lock, L.F.; Lee, A.; Buchberg, A.M.; et al. Chromosomal localization of seven members of the murine TGF- $\beta$ superfamily suggests close linkage to several morphogenetic mutant loci. Genomics 1990, 6, 505-520. [CrossRef] 
32. Li, D.; Sakuma, R.; Vakili, N.A.; Mo, R.; Puviindran, V.; Deimling, S.; Zhang, X.; Hopyan, S.; Hui, C. Formation of Proximal and Anterior Limb Skeleton Requires Early Function of Irx3 and Irx 5 and Is Negatively Regulated by Shh Signaling. Dev. Cell 2014, 29, 233-240. [CrossRef]

33. Michos, O.; Panman, L.; Vintersten, K.; Beier, K.; Zeller, R.; Zuniga, A. Gremlin-mediated BMP antagonism induces the epithelial-mesenchymal feedback signaling controlling metanephric kidney and limb organogenesis. Development 2004, 131, 3401-3410. [CrossRef] [PubMed]

34. Riddle, R.D.; Johnson, R.L.; Laufer, E.; Tabin, C. Sonic hedgehog mediates the polarizing activity of the ZPA. Cell 1993, 75, 1401-1416. [CrossRef]

35. Kuijper, S.; Feitsma, H.; Sheth, R.; Korving, J.; Reijnen, M.; Meijlink, F. Function and regulation of Alx4 in limb development: Complex genetic interactions with Gli3 and Shh. Dev. Biol 2005, 285, 533-544. [CrossRef]

36. Farin, H.F.; Lüdtke, T.H.-W.; Schmidt, M.K.; Placzko, S.; Schuster-Gossler, K.; Petry, M.; Christoffels, V.M.; Kispert, A. Tbx2 terminates shh/fgf signaling in the developing mouse limb bud by direct repression of gremlin1. PLoS Genet. 2013, 9, e1003467. [CrossRef] [PubMed]

37. Knezevic, V.; De Santo, R.; Schughart, K.; Huffstadt, U.; Chiang, C.; Mahon, K.A.; Mackem, S. Hoxd-12 differentially affects preaxial and postaxial chondrogenic branches in the limb and regulates Sonic hedgehog in a positive feedback loop. Development 1997, 124, 4523.

38. Mercader, N.; Selleri, L.; Criado, L.M.; Pallares, P.; Parras, C.; Cleary, M.L.; Torres, M. Ectopic Meis1 expression in the mouse limb bud alters P-D patterning in a Pbx1-independent manner. Int J. Dev. Biol. 2009, 53, 1483-1494. [CrossRef]

39. Haack, H.; Gruss, P. The Establishment of Murine Hox-1 Expression Domains during Patterning of the Limb. Dev. Biol. 1993, 157, 410-422. [CrossRef]

40. Lu, P.; Yu, Y.; Perdue, Y.; Werb, Z. The apical ectodermal ridge is a timer for generating distal limb progenitors. Development 2008, 135, 1395. [CrossRef]

41. Cobb, J.; Dierich, A.; Huss-Garcia, Y.; Duboule, D. A mouse model for human short-stature syndromes identifies Shox2 as an upstream regulator of Runx2 during long-bone development. Proc. Natl. Acad. Sci. USA 2006, 103, 4511. [CrossRef] [PubMed]

42. Davis, A.P.; Witte, D.P.; Hsieh-Li, H.M.; Potter, S.S.; Capecchi, M.R. Absence of radius and ulna in mice lacking hoxa-11 and hoxd-11. Nature 1995, 375, 791-795. [CrossRef] [PubMed]

43. Kherdjemil, Y.; Lalonde, R.L.; Sheth, R.; Dumouchel, A.; de Martino, G.; Pineault, K.M.; Wellik, D.M.; Stadler, H.S.; Akimenko, M.-A.; Kmita, M. Evolution of Hoxa11 regulation in vertebrates is linked to the pentadactyl state. Nature 2016, 539, 89-92. [CrossRef] [PubMed]

44. Woltering, J.M.; Noordermeer, D.; Leleu, M.; Duboule, D. Conservation and Divergence of Regulatory Strategies at Hox Loci and the Origin of Tetrapod Digits. PLoS Biol. 2014, 12, e1001773. [CrossRef]

45. Sheth, R.; Bastida, M.F.; Kmita, M.; Ros, M. "Self-regulation," a new facet of Hox genes' function. Dev. Dyn. 2014, 243, 182-191. [CrossRef]

46. Mayan, M.D.; Carpintero-Fernandez, P.; Gago-Fuentes, R.; Martinez-de-Ilarduya, O.; Wang, H.-Z.; Valiunas, V.; Brink, P.; Blanco, F.J. Human articular chondrocytes express multiple gap junction proteins: Differential expression of connexins in normal and osteoarthritic cartilage. Am. J. Pathol 2013, 182, 1337-1346. [CrossRef]

47. Zhao, F.; Bosserhoff, A.-K.; Buettner, R.; Moser, M. A Heart-Hand Syndrome Gene: Tfap2b Plays a Critical Role in the Development and Remodeling of Mouse Ductus Arteriosus and Limb Patterning. PLoS ONE 2011, 6, e22908. [CrossRef]

Publisher's Note: MDPI stays neutral with regard to jurisdictional claims in published maps and institutional affiliations.

(C) 2020 by the authors. Licensee MDPI, Basel, Switzerland. This article is an open access article distributed under the terms and conditions of the Creative Commons Attribution (CC BY) license (http://creativecommons.org/licenses/by/4.0/). 